\title{
La Economía Social: una vía para la creación de un empleo estable y de calidad
}

\author{
Aida Llamosas Trapaga \\ Doctora en Derecho por la Universidad de Deusto \\ Profesora de Derecho del Trabajo y de la Seguridad Social
}

Sumario: I. Concepto de Economía Social. II. La Economía Social en España. III. Crisis y economía social. IV. La Economía Social como respuesta para un nuevo modelo de empleo. V. Bibliografía.

Resumen: El concepto y las entidades de Economía Social no son nuevos, sino más bien lo contrario, pero la situación actual está demostrando que nos encontramos en un momento vital para decidir que caminos debemos tomar. La crisis ha remitido y junto con el crecimiento se está volviendo a la creación de empleo. La cuestión reside en el tipo de empleo que estamos creando y en el impacto que eso está suponiendo tanto para las personas como para la sociedad en su conjunto. Las empresas reclaman una mayor flexibilidad, y aun reconociendo la necesidad de la misma es necesario encontrar la fórmula para que ello no sea un obstáculo en la consecución de un empleo estable y de calidad.

Palabras clave: economía social, valores, empleo y estabilidad.

Abstract: The concept and Social Economy entities are not new, but rather the opposite, but the current situation is proving that we are at a vital time to decide that roads must take. The crisis has sent and together with growth is returning to employment creation. The companies are demanding greater flexibility and even recognizing the need it is necessary to find the way so that it is not an obstacle in the achievement of a stable job and quality.

Keywords: Social economy, principles, employment and stability. 


\section{Concepto de Economía Social}

En los últimos años se hace referencia al término de Economía Social como un concepto innovador, sin embargo, se trata de una concepción con una larga tradición y una notable historia.

El asociacionismo y el cooperativismo histórico han sido los dos elementos que se han constituido como el eje vertebrador de esta nueva forma de entender la economía.

Las sociedades de resistencia y las sociedades de socorros mutuos suponen el antecedente y reflejan la necesidad de los trabajadores de encontrar apoyo en un sistema capitalista industrial cuyo único objetivo era el de la producción y la obtención de mayores beneficios, sin reparar en las condiciones de trabajo y vida de las clases menos favorecidas.

Los diversos movimientos obreros dejaron clara constancia de que el sistema económico imperante debía cambiar, y con ello la visión de los titulares de los medios de producción que no podían seguir viendo en los trabajadores unas meras herramientas para la consecución de los intereses.

Resulta especialmente relevante, la creación, en el año 1844, de la Rochdale Equitable Pioneers Society, una cooperativa de consumo, que si bien no fue la primera, si supuso la formación de las bases para el cooperativismo moderno, estableciendo en su organización una serie de principios que se mantienen hasta el día de hoy en el movimiento cooperativista, gracias a la Alianza Cooperativa Internacional, y que suponen una verdadera declaración de intenciones en lo que se refiere a la forma de entender la empresa frente a los postulados capitalistas.

Tal y como se mencionaba previamente, el concepto de la Economía Social no es nuevo, y en lo que se refiere a la literatura económica, la primera referencia puede situarse en el año 1830, cuando el economista liberal francés DUNOYER publicó su Nouveau traité d'économie sociale, en el que lleva a cabo una aproximación moral de la economía'.

En este momento comenzó a tomar forma una nueva corriente en el pensamiento económico, basándose en los análisis llevados a cabo por MALTHUS y SISMONDI, que focalizaban su atención en los fallos del propio mercado y en los desequilibrios que esos fallos podían provocar.

Estos autores supieron dar una nueva visión a la Economía Social, haciendo referencia a las organizaciones innovativas creadas para dar una respuesta a las cuestiones generadas por el capitalismo.

\footnotetext{
1 DunOYer, C.: Nouveau traité d'économie sociale, Imprimerie Fournier, Paris, 1830.
} 
SISMONDI' ${ }^{2}$ entendía que el auténtico objeto de la Economía Social no era la riqueza, sino el individuo, el ser humano, y que por tanto este tenía que ostentar el protagonismo.

Aunque resulta cierto que esta nueva corriente de pensamiento realizó notables esfuerzos, resulta especialmente relevante el hecho de que pusieran de manifiesto las anomalías presentes en el sistema económico y las consecuencias que estas podían tener. Sin embargo, estas teorizaciones no fueron respaldadas por acciones o políticas concretas que pudiesen ofrecer una alternativa válida para dar paso a un nuevo sistema.

Entendía WALRAS que la Economía Social no era un simple concepto, sino que debía ser considerada como una parte esencial, fundamental, de la ciencia económica y la justicia social el objetivo prioritario de la misma3.

Este mismo autor enfatizaba el papel fundamental de las cooperativas en la resolución de los conflictos sociales atendiendo a su función económica, consistente en la limitación del desarrollo del capitalismo y de su función moral en base a la capacidad de democratización de los procesos productivos.

A este respecto cabe recordar la obra de MILL Principles of Political Economy 4 donde se focalizaba la atención en el asociacionismo entre los trabajadores, ya sea en forma de cooperativas o de mutua, y se exhorta a la promoción de este tipo de empresas por las ventajas económicas, éticas y morales que son capaces de generar.

Desde esta perspectiva, es necesario recordar que a pesar del amplio recorrido histórico de la Economía social, es únicamente a partir de los años 70 cuando se ha producido un renacimiento de este sector.

En efecto, la promoción de la Economía Social queda ligada a la propia crisis económica vivida en aquellos años, algo ciertamente muy parecido a lo que está ocurriendo en estos momentos.

La virulencia de la crisis económica pasada, la fuerte ralentización de la economía, y sobre todo las alarmantes tasas de desempleo, junto con cada vez una mayor externalización de las actividades productivas, han hecho que surjan nuevas dinámicas de cooperación entre los actores económicos.

También resulta cierto, que este nuevo despertar ha sido desigual, o entendido de diversas formas en los diferentes países, así como

2 Simonde de SISMOndi, J.C.L.: Nuevos principios de economía política. O de la riqueza en sus relaciones con la población, Editorial Icaria, Barcelona, 2016. (Obra original 1827).

3 WALRAS, L.: Études d'économie sociale, Dentu, Paris, 1896.

4 Stuart Mill, J.: Principles of Political Economy with some of their Applications to Social Philosophy, Hackett Publishing, Indianapolis, 2004. (Obra original,1848). 
el propio concepto de la Economía Social, su alcance y el apoyo a la misma a través de políticas de carácter público.

La identificación de la Economía Social, tal y como es conocida a día de hoy, tiene su referente en Francia, alrededor de los años 70 del pasado siglo XX, cuando se produce la constitución, a cargo de la representación de las cooperativas, las mutuas y las asociaciones, del Comité National de Liaison des Activités Cooperatives, Mutuelles et Associatives.

Dicho Comité publicó la Charte de l'Economie Sociale ${ }^{5}$ donde la economía social fue definida como el conjunto de organizaciones, no pertenecientes al sector público donde se gestiona, de forma democrática, asegurando, de forma paritaria, los derechos y los deberes de los socios, atendiendo a un particular sistema en el régimen de propiedad y en la distribución de los beneficios, destinando los mismos al desarrollo de la propia organización y a la mejora de los servicios ofrecidos a la sociedad 6 .

Es a partir del año 1989, cuando la Comisión Europea comienza a llevar a cabo iniciativas para promover la economía social, publicando algunas obras que estudiaban las empresas de economía social y la creación del mercado europeo sin fronteras.

Poco a poco se fue avanzando y ya en el año 1990, en el seno del Parlamento europeo se comienza a trabajar el intergrupo de Economía Social, elaborando diversas propuestas en esta materia con más o menos éxito en su aplicación.

Hasta la aprobación de la Ley española sobre economía social, la caracterización más reciente de este ámbito podía encontrarse en la Carta de los principios de la economía social del 2002, que fue redactada por la Conferencia Europea permanente de las cooperativas, mutuas, asociaciones y fundaciones, esto es, CEP-CMAF.

En dicho documento se matizan una serie de principios que resultan fundamentales:

1. La primacía de la persona y de los fines sociales sobre el capital;

2. La adhesión y la participación libre y voluntaria;

3. El control democrático por parte de los socios;

4. La conciliación de los intereses del socio con los intereses generales;

5 Charte de l'Economie Sociale. Comité National de Liaison des Activités Cooperatives, Mutuelles et Associatives, 1980.

6 Moreau, J.: Essai sur une politique de l'Economie Sociale, du troisième secteur vécu au troisième secteur voulu, C.I.E.M, Paris, 1982, p. 91. 
5. El respeto a los principios de responsabilidad y solidaridad;

6. La gestión autónoma e independiente respecto a las autoridades de carácter público;

7. La destinación de los beneficios al desarrollo y consecución de los fines.

\section{La Economía Social en España}

En el ordenamiento jurídico español el concepto de economía social comienza a asumir relevancia jurídica con la aprobación de la Ley 31/1990 de Presupuestos Generales del Estado ${ }^{7}$ y con la creación del Instituto Nacional de Fomento de la Economía Social-INFES.

Se trataba de un ente, de carácter administrativo, que venía a sustituir a la Dirección de Cooperativas y Sociedades Laborales, ubicándose al interno del Ministerio de Trabajo y de Seguridad Social.

Finalmente, en el año 2011 se produce la aprobación de la Ley 5/2011 sobre Economía Social ${ }^{8}$.

Con la aprobación de dicha normativa el legislador español se situó a la vanguardia del reconocimiento legislativo de esta materia, siendo un auténtico precursor.

En este sentido es necesario recordar que España no es en absoluto ajena a esta materia, puesto que ya en el año 1978 y con la aprobación de la Carta Magna, quiso incluir entre sus preceptos una llamada de atención al propio legislador, a través de su artículo 129, en el que reconoce que insta a los poderes públicos a promover «eficazmente las diversas formas de participación en la empresa» fomentando "mediante una legislación adecuada, las sociedades cooperativas» estableciendo «los medios que faciliten el acceso de los trabajadores a las propiedad de los medios de producción ${ }^{9}$.

Puede decirse por tanto que por parte de las autoridades españolas existe un inequívoco empeño en favorecer las organizaciones de la economía social, promoviendo la relación entre estas y las autoridades públicas.

Pero, atendiendo estrictamente a la Ley española, ¿cuál es el verdadero objetivo de la misma?

7 Ley 31/1990, de 27 de diciembre, de Presupuestos Generales del Estado para 1991. BOE de 28 de diciembre de 1990. Núm. 311.

8 Ley 5/2011, de 29 de marzo, de Economía Social. BOE de 30 de marzo de 2011. Núm. 76.

9 Constitución Española. BOE de 29 de diciembre de 1978. Núm. 311. 
Tanto el preámbulo de la misma como su artículo primero lo indican, mencionando que la finalidad no es otra que la de instaurar un marco jurídico, que sustituyendo las normativas vigentes de cada uno de los entes que componen la economía social, suponga el reconocimiento y una mayor visibilidad de este ámbito, definiendo que se entiende por tal y concretando una serie de principios.

Son por tanto tres los elementos a poner de manifiesto:

1. La elaboración de un concepto jurídico basado en los valores y los principios de la economía social, cuyo objetivo es:

a) el reconocimiento jurídico de la economía social y de las entidades que forman parte de ella;

b) la visibilidad institucional de la economía social;

c) una mayor certeza jurídica para este ámbito.

2. El reconocimiento a los empresarios de la economía social de su derecho a constituir asociaciones para la representación y la tutela de sus propios intereses;

3. El reconocimiento de la promoción de la economía social como una actividad de interés general.

En lo que se refiere a la estructura del propio texto normativo, la Ley queda compuesta por 9 artículos, 7 disposiciones adicionales, dos disposiciones transitorias y cuatro disposiciones finales.

Como ya ha sido mencionado previamente el artículo primero habla del objetivo de la normativa, mientras que el artículo 2 hace referencia al concepto de la economía social. Una definición esta de la cual pueden extraerse algunas características comunes respecto de los entes que forman parte de la economía social:

1. Se hace referencia a entes de naturaleza privada, instituciones que no forman parte del sector público, y que por tanto, quedan fuera del alcance de su control;

2. Son entes con personalidad jurídica autónoma;

3. Desde un punto de vista formal son organizaciones con plena capacidad y libertad de decisión respecto a la gestión y al control de la propia actividad;

4. Se fundamentan en el principio de la adhesión voluntaria;

5. La eventual distribución de los beneficios viene dada en base al trabajo llevado a cabo y no en función del capital aportado por cada uno de ellos;

6. Son organizaciones democráticas con un principio fundamental «una persona, un voto». 
Pero quizás es el artículo 4 el más significativo, ya que en el mismo se recogen los principios guía, reconocimiento como tales los siguientes:

1. La primacía de las personas y del fin social respecto del capital. Este principio se concreta en una gestión independiente, transparente, democrática y participativa, lo que a su vez supone dar prioridad a las personas en los procesos decisionales, atendiendo al trabajo llevado a cabo por estos y no a su aportación en términos de capital.

A través de este principio el legislador subraya desde un primer momento cual es el principio fundamental: la primacía de la persona y de su esfuerzo sobre el capital.

Se trata, sin duda alguna, de un principio que distingue que forma nítida las entidades de la economía social en comparación con otro tipo de empresas u organizaciones, en las cuales el objetivo no es otro que la maximización del beneficio para su posterior distribución a los socios en favor de su aportación dineraria.

Atendiendo, al papel central que se concede en favor de la persona, también se solicita que la misma participe, de forma directa en el proceso decisional de la entidad.

A diferencia de lo que ocurre en otro tipo de sociedades, en las cuales la gestión se orienta a la generación de beneficios para la posterior retribución a los socios, que tomaran las decisiones en función del capital aportado y siempre con la finalidad de obtener mayores ganancias, en las empresas de economía social la gestión de las misma queda supeditado al servicio a las personas, a la consecución de objetivos sociales en beneficio de los socios o de terceras personas, tomando decisiones de forma democrática y repartiendo los beneficios de forma equitativa.

2. La distribución de los beneficios generados en función de la prestación laboral o de servicios de cada uno de los socios.

Este principio supone un esfuerzo llevado a cabo por el legislador para ligar este tipo de actividades a valores tales como:
a) la remuneración de la actividad laboral de los socios preva- lece respecto a la remuneración del capital:
b) reinversión e investigación para mejorar los servicios ofreci- dos y reducir sus costes;
c) utilización de los beneficios para acciones de carácter social;
d) promoción del asociacionismo y de la acción social. 
3. Promoción del principio de solidaridad, al interno de las propias entidades y respecto de la propia comunidad, favoreciendo el desarrollo local, la igualdad de oportunidades entre hombres y mujeres, la cohesión social, la inclusión de las personas en riesgo de exclusión social, una ocupación estable y de calidad, la conciliación de la vida laboral y familiar y la sostenibilidad.

Es necesario tener en cuenta que las entidades de economía social no actúan en un entorno cerrado, sino en el ámbito de una sociedad, por lo que deben afrontar, de forma constante cambios y desafíos de carácter social.

Y es precisamente por ello, que la economía social promueve una serie de valores y de principios que resultan necesarios para una sociedad más justa, favoreciendo la solidaridad, el esfuerzo, la igualdad, el desarrollo local, la ocupación, la calidad, la sostenibilidad, etc.

4. La independencia respecto de los poderes públicos.

La independencia respecto del sector público garantiza la libertad de estas entidades para llevar a cabo su gestión en la forma que estimen más conveniente, para definir sus propios objetivos, la conformación de sus órganos internos, así como su funcionamiento, etc.

\section{Crisis y economía social}

La situación económica actual y la necesidad de apoyarse en nuevos paradigmas, ha propiciado que la Economía Social, si bien a lo largo de los últimos años ha tenido una creciente importancia, se sitúe, de nuevo como uno de los mecanismos más importantes en la creación de nuevas vías para entender el modelo económico.

La crisis económica en la que el mundo se ha visto inmerso desde prácticamente una década, ha provocado la quiebra de numerosas empresas, una tasa de desempleo desorbitada en nuestro país, arrojando datos especialmente duros respecto de los colectivos más vulnerables, un crecimiento sin precedentes de la pobreza y un aumento más que notable de las desigualdades.

Actualmente nos encontramos en la consolidación del proceso de recuperación y España ha superado todas las previsiones, incluso las más optimistas en lo que se refiere el crecimiento, arrojando unos datos ciertamente muy positivos que se sitúan en el 3,2\%. 
Sin embargo, y a pesar de que en lo que al crecimiento se refiere no solo se cumplieron los mejores pronósticos, sino que se mejoraron, no puede decirse lo mismo del mercado laboral.

En el primer trimestre de 2017 la tasa de desempleo se situó en $18,2 \%$ y puede apreciarse que se produjo un descenso no solo en la cifra total sino en todos los colectivos, esto es, se ha producido un descenso en la tasa de desempleo relativa a los hombres, las mujeres y menores de 25 años $^{10}$.

Según datos del Ministerio de Empleo y Seguridad Social, el pasado año finalizó con una reducción del desempleo histórica, registrando un descenso de 390.534 personas, recuperando de ese modo la mitad del empleo destruido durante la crisis, y siendo, tras Alemania el país que más empleo está creando en la eurozona.

Efectivamente, estos datos son positivos y demuestran que España está saliendo, de manera bastante rápida de la crisis, pero la pregunta que cabe hacerse es ¿cuál es el coste de esta recuperación?

Si bien resulta cierto que se está creando empleo, es necesario observar que tipo de empleo es, puesto que aunque se trata de una noticia positiva en términos generales, si se examina con mayor detenimiento puede observarse que las nuevas ocupaciones, en su mayoría, carecen de calidad y estabilidad.

Un mercado laboral que crece con una base inestable como la actual no puede avanzar hacia un futuro en el que se pueda hacer frente a los nuevos desafíos tecnológicos y económicos.

Es necesario avanzar hacia un modelo de relaciones laborales que focalice su atención en el ser humano.

Los datos disponibles han puesto de manifiesto una realidad incuestionable, las empresas de economía social, sin duda alguna, han sufrido los efectos de la recesión económica, y han recorrido un camino lleno de dificultades ante la adversa situación, pero dicho impacto ha sido menor que en el resto de las empresas, y las entidades de economía social han sabido reaccionar de mejor manera, siendo de ese modo, también más rápida su recuperación.

Siendo así, han sido muchos los que han focalizado su atención en este fenómeno para comprender que es lo que hace que las empresas de la economía social sean diferentes de las empresas capitalistas propiamente.

10 Instituto Nacional de Estadística. Encuesta Población Activa. 2017. 
No existe una única razón o receta que explique esta mayor resistencia de la economía social a la crisis, sino que son varios los elementos a analizar.

En numerosas ocasiones se produce una desinformación entre los accionistas o las personas encargadas de tomar decisiones en la empresa y la verdadera situación de la misma.

Son los trabajadores los que observan los problemas y las dificultades a las que día a día se tienen que enfrentar, pero en muchas ocasiones las decisiones que se toman no tienen en cuenta esa perspectiva.

En las empresas de economía social se produce un acercamiento entre el proceso decisional y los propios trabajadores, por lo que los órganos de decisión cuentan con una información mayor y más cercana a la realidad de todos los sujetos involucrados, lo que supone una individualización mayor de los problemas.

Cuando resulta posible determinar de forma clara cuales son los problemas concretos a los que se enfrenta una empresa, también resulta más fácil encontrar mejores soluciones.

Además, la responsabilidad que se les atribuye a los trabajadores en la toma de decisiones para mejorar la empresa hace que los mismos sientan la misma como una parte importante de sí mismos, un lugar en el que no solo llevan a cabo su prestación de trabajo sino en el que se les toma en consideración dando peso a su opinión y sus acciones, sin verlos como una mera herramienta para la consecución de beneficios.

Asimismo, este proceso de codecisión hace que exista una mayor flexibilidad en la empresa lo que de forma general resulta muy favorable, especialmente en aquellos periodos económicos de incertidumbre.

Es necesaria la introducción de una mayor flexibilidad en los mercados, y sobre todo en las empresas para que estas últimas puedan competir en un mercado que se torna cada vez más agresivo y competitivo.

Ciertamente, la flexibilidad hace que ante determinados problemas puedan encontrarse soluciones que resulten menos drásticas, llevando a cabo ajustes que supongan un efecto menos negativo de lo que supondría en un contexto de mayor rigidez.

El problema es que si bien resulta necesaria una mayor flexibilidad, eso en numerosas ocasiones se ha traducido en una merma de los derechos de los trabajadores y una reducción de las condiciones de trabajo.

Por lo que hay que encontrar un punto de equilibrio entre esta ansiada flexibilidad y un empleo de calidad y estable. 
El trabajador, como no podría de ser de otra manera, busca una estabilidad en su empleo, en primer lugar porque el mismo representa la base de su supervivencia y además, porque eso le supone una mayor protección frente a posibles injerencias o decisiones arbitrarias del empresario.

Por su parte, el empresario se debate entre dos frentes.

Normalmente contar con trabajadores estables suele suponer que los mismos tengan un conocimiento mayor de la empresa, de su cultura y de su funcionamiento, y al mismo tiempo, eso supone una mayor formación, lo que redunda en un beneficio para la empresa.

Pero por el contrario, la estabilidad en los empleos hace que la empresa se pregunte si eso no supone un freno en el caso que haya que acometer cambios para adaptarse a nuevas situaciones. Una duda que se ha hecho cada vez más evidente, si tenemos en cuenta que los periodos de incertidumbre se han convertido en algo habitual en los mercados económicos.

Cierto es que el deber y el derecho a trabajar se recoge como uno de los preceptos de nuestra Carta Magna, por lo que como indican los autores Martín Valverde, Rodríguez Sañudo y García Murcia «el derecho al trabajo es el punto de apoyo constitucional del principio de estabilidad en el empleo» ${ }^{11}$.

Sin embargo, el artículo $35^{12}$ no dispone nada más acerca de ese trabajo, de su contenido y sus características.

No obstante, y a pesar de que la normativa no nos indica nada en ese sentido, el Tribunal Constitucional si ha entrado a conocer dicha cuestión reconociendo dos ámbitos, uno de carácter individual y otro de carácter colectivo.

En lo que se refiere al aspecto individual, el mismo se concreta en el derecho que asiste a toda persona a acceder a un puesto de trabajo concreto si cuenta con los requisitos para ocupar el mismo, así como a tener continuidad y estabilidad en el mismo, si no existe una causa justa para que deba abandonarlo.

En lo referente al aspecto colectivo, resulta más una llamada de atención a los poderes públicos, para que los mismos a través de sus políticas lleven a cabo acciones que conduzcan hacia el pleno empleo, tal y como se recoge en el propio artículo 40 de la Constitución.

Por tanto si bien el objetivo del principio de estabilidad pretende garantizar en favor del trabajador una continuidad, eso no significa

11 Martín Valverde, A; Rodríguez Sañudo, F. y García Murcia, J.: Derecho del Trabajo. Editorial Tecnos, Madrid, 2009, 18. ${ }^{a}$ ed., pp. 147.

12 Artículo 35 de la Constitución Española. 
que el mismo no pueda sufrir algún tipo de cambio al interno de la empresa, garantizando de ese modo la estabilidad y al mismo tiempo la flexibilidad.

\section{La Economía Social como respuesta para un nuevo modelo de empleo}

La búsqueda del equilibrio entre la estabilidad y la flexibilidad sufrió un duro revés con la reforma laboral llevada a cabo en el año 2012, a través de la Ley $3 / 2012^{13}$.

En su preámbulo la normativa señala que el objetivo de la modificación no es otro que el de tratar de garantizar la flexibilidad en favor del empresario, para que este pueda organizar los recursos de la empresa, incluidos los recursos humanos, en función de las necesidades especificas, pero protegiendo, al mismo tiempo, la seguridad de los trabajadores respecto de sus puestos de trabajo.

Se entiende por tanto que los nuevos preceptos tratan de equilibrar los intereses tanto de una parte como de la otra, entendiendo la flexibilidad como la solución alternativa a la destrucción del empleo.

Se presume que si el empresario, puede hacer uso de una mayor flexibilidad, adecuando los recursos existentes a las nuevas circunstancias, no optará por decisiones más drásticas.

Además, puede suponer una oportunidad para avanzar en la conciliación de la vida laboral y personal.

Esa flexibilidad exige menores rigideces respecto al despido, pero al mismo tiempo una mayor protección social para las personas que puedan quedar en situación de desempleo y sobre todo mayores facilidades para llevar a cabo nuevos contratos.

Será necesario por tanto, instaurar estrategias globales de formación para los trabajadores, de modo que posean una alta empleabilidad y adaptabilidad, especialmente respecto de aquellos colectivos que pueden resultar más vulnerables o más expuesto a dichos cambios.

Y no solo, sino que además debe tenderse a políticas activas de empleo eficaces que puedan de forma rápida reducir, al máximo posible, los periodos de desempleo, facilitando que los trabajadores puedan moverse de un puesto de trabajo a otro, sin que por ello pierdan sus derechos.

13 Ley $3 / 2012$ de 6 de julio, de medidas urgentes para la reforma del mercado laboral. BOE de 7 de julio de 2012. Núm. 162. 
La cuestión es que la Ley 3/2012 parece centrarse únicamente en el ámbito de la flexibilidad, olvidando los mecanismos necesarios para la creación y el mantenimiento de un empleo digno y de calidad, que ponga como punto de referencia al trabajador, y no los meros beneficios económicos.

La introducción de mecanismos para facilitar el despido no vinieron acompañados de políticas para la protección de social de los trabajadores, por lo que la balanza se desequilibró hacía el lado de la flexibilidad, permitiendo un despido, no solo más fácil si no más barato, sin ofrecer a los trabajadores los elementos necesarios y eficaces para encontrar un nuevo empleo.

Esta situación ha hecho que se vuelva la mirada hacia las empresas de economía social.

Estas empresas han demostrado una mayor resistencia hacia periodos adversos y eso, se debe a su especial distribución de beneficios, estableciendo políticas a largo plazo que faculta que posean mayores reservas que no vienen distribuidas entre los socios.

El especial funcionamiento interno de las empresas de economía social hace que los socios, tomando decisiones de forma conjunta puedan adecuar sus salarios, evitando de ese modo alterar los niveles de empleo.

Los recursos para la producción se asignan de manera más inteligente. La solidaridad entre trabajadores, su especial vinculación al organismo y la confrontación de ideas entre los propios socios promueve que los empleados estén dispuestos a mayores sacrificios, puesto que comprenden que dichos sacrificios redundaran en su propio beneficio, permitiendo, al mismo tiempo una estabilidad en la empresa que no se da en las empresas puramente capitalistas.

En numerosas ocasiones las crisis económicas han sido revulsivo para la creación de este tipo de empresas o para la conversión de empresas capitalistas, en las que los trabajadores, y ante la gravedad de la situación económica y el temor de perder su empleo han optado por reconvertirlas en cooperativas.

Pero las entidades de economía social con sus valores y principios no solo permiten el pleno desarrollo de las personas y de su entorno, sino que además se han convertido en una herramienta eficaz en la lucha contra la pobreza y la exclusión social.

En una visión muy simplista, pero no por ello menos cierta, puede decirse que las empresas capitalistas focalizan su atención en el crecimiento, mientras que las empresas de economía social se centran en el desarrollo.

Resulta necesario poner de manifiesto que cuando se hace referencia al término crecimiento, esto no significa lo mismo que desarrollo. 
SCHUMPETER fue uno de los primeros economistas en diferenciar ambos términos, y en el año 1912, en su obra Teoría del Desarrollo Económico, definió el crecimiento como un simple aumento de la riqueza, mientras que por desarrollo entendía una serie de factores sociales, culturales, políticos y psicológicos fruto de un proceso de innovación tecnológica impulsada por la creatividad de los empresarios ${ }^{14}$.

Por ello, es necesario remarcar que crecimiento económico no tiene por qué significar, necesariamente desarrollo.

Ciertamente, de forma habitual el crecimiento ha sido definido como una expansión continua, a lo largo del tiempo, de la actividad económica, y más concretamente de la producción nacional de un concreto país.

Siendo así, para proceder a la medición de dicho crecimiento la herramienta más usada ha sido el PIB ${ }^{15}$.

El desarrollo económico por su parte, puede definirse como la capacidad para crear riqueza con el objetivo de mantener o promover el bienestar económico y social.

Se trata de un proceso a través del cual una sociedad moderniza su organización económica, permitiendo de ese modo un crecimiento de forma continuada, propiciando transformaciones sociales e incrementando la calidad de vida de las personas.

Es necesario pensar en el crecimiento, pero en un crecimiento que es capaz de generar empleo, un crecimiento que permita a las personas tener un modo de vida, propiciando la libertad de las mismas, distribuyendo los beneficios de forma equitativa en favor de la cohesión social ${ }^{16}$.

14 SChumpeter, J.A.: Teoría del desarrollo económico, FCE. México, 1976.

15 A pesar de que el PIB suele ser la herramienta más utilizada, son muchos los autores que indican que este sistema posee muchas limitaciones para medir el progreso y el desarrollo de un país, puesto que solo abarca parámetros económicos. Mencionan esta cuestión los autores BECCHETt y CeRMelLI, que a través de las opiniones de diversos autores ponen en evidencias las carencias que presenta el PIB como herramienta. BECCHETTI, L. y CeRMELLI, M.: «Reduccionismos económicos y voto con la cartera», Revista de Fomento Social, núm. 69, 2014.

16 «Una sociedad como la capitalista, que en apariencia, da riendas sueltas a la libertad, provoca que su despliegue ilimitado obstruya, impida, ahogue, su posible extensión a toda la sociedad. La libertad como valor tiene un límite lógico: la afectación de la libertad de otros. Más allá de ese límite, se convierte en su contrario, en un antivalor. Sobrepasado ese umbral, mientras más libre sea un sujeto determinado para moverse por la compleja red de relaciones social, menos movilidad y menos libertad tendrán otros. Evitarlo sólo sería posible si se colocara un valor distinto por encima de la libertad misma. Pero no es eso lo que ocurre en la sociedad del capital. Lo cotidiano allí es que la excesiva libertad de unos implique la ausencia de liber- 
La desigualdad entre pobres y ricos se hace cada vez más evidente y la clase media se ha posicionado en una situación realmente muy vulnerable, con muchos de sus miembros en grave riesgo de exclusión social ${ }^{17}$.

La lucha contra la pobreza y la exclusión social comienza con un empleo estable, digno y con una remuneración justa, porque tal y como indican algunos autores "no solo confiere independencia a las personas, sino también dignidad, participación en la sociedad y acceso a los servicios y prestaciones» ${ }^{18}$.

La economía social ha logrado convertirse en un mecanismo eficaz para luchar contra esta desigualdad y exclusión apoyando a las personas más desfavorecidas.

La relevancia de las entidades de la economía social así como las bondades de su especial forma de entender la empresa y la economía han hecho que desde la Unión Europea los agentes políticos, económicos y sociales traten de avanzar, a través de diversas iniciativas, en el reconocimiento, desarrollo y promoción de la misma.

Así, en el año 2010 la Unión Europea, que a lo largo de muchos años ha llevado a cabo diversas iniciativas en esta materia, decidió poner en marcha Europa 2020, una estrategia para el empleo y el crecimiento integrador, sostenible e inteligente.

Si a lo largo de los años la economía social ha contribuido a la creación de Europa, no cabe duda de que lo seguirá haciendo a través de esta nueva estrategia.

tad de otros. Y eso es, precisamente, la injusticia. El mundo capitalista se ha movido hasta ahora bajo la consigna engañosa de la libertad y, por eso, ha traído tanta injusticia» FAbelo Corzo, J.R.: Los valores y sus desafíos actuales, Libros en Red. 2004. p. 137.

17 Resulta interesante a este respecto la reflexión que hace el Papa Benedicto XVI cuando menciona que «la dignidad de la persona y las exigencias de la justicia requieren, sobre todo hoy, que las opciones económicas no hagan aumentar de manera excesiva y moralmente inaceptable las desigualdades y que se siga buscando como prioridad el objetivo del acceso al trabajo por parte de todos, o que lo mantengan. Pensándolo bien, esto es también una exigencia de la "razón económica". El aumento sistémico de las desigualdades entre grupos sociales dentro de un mismo país y entre las poblaciones de los diferentes países, es decir, el aumento masivo de la pobreza relativa, no sólo tiende a erosionar la cohesión social y, de este modo, poner en peligro la democracia, sino que tiene también un impacto negativo en el plano económico por el progresivo desgaste del "capital social", es decir, del conjunto de relaciones de confianza, fiabilidad y respeto de las normas, que son indispensables en toda convivencia civil» Encíclica Caritas in Veritate. 29 de junio del 2009.

18 La economía social en la Unión Europea. Comité Económico y Social Europeo. 2012, p. 94. 
La solidaridad, el conocimiento, la innovación y los valores son las señas de identidad de esta nueva forma de hacer economía en la que los derechos y las necesidades de las personas se encuentran por encima del capital y en la que la particular distribución de los beneficios contribuye a la búsqueda de una mayor calidad en el empleo, combatiendo la inestabilidad laboral y ofreciendo oportunidades de trabajo que permiten la lucha contra la exclusión social.

La economía social ofrece soluciones a los retos económicos y sociales que se están planteando en la sociedad actual, aplicando unos principios y unos valores que buscan un empleo de calidad que vuelva a poner a la persona como centro de todas sus actividades.

\section{Bibliografía}

BeCChetTI, L. y Cermell, M.: «Reduccionismos económicos y voto con la cartera», Revista de Fomento Social, núm. 69, 2014.

CARITAS in Veritate. 29 de junio del 2009.

CharTe de I'Economie Sociale. Comité National de Liaison des Activités Cooperatives, Mutuelles et Associatives, 1980.

Constitución Española. BOE de 29 de diciembre de 1978. Núm. 311.

DUNOYER, ChARLES.: Nouveau traité d'économie sociale. Imprimerie Fournier, Paris, 1830.

Fabelo Corzo, J.R.: Los valores y sus desafíos actuales, Libros en Red, 2004.

InstiTUTo Nacional de Estadística. Encuesta Población Activa. 2017. La economía social en la Unión Europea. Comité Económico y Social Europeo. 2012.

LeY 5/2011, de 29 de marzo, de Economía Social. BOE de 30 de marzo de 2011. Núm. 76.

LEY 3/2012 de 6 de julio, de medidas urgentes para la reforma del mercado laboral. BOE de 7 de julio de 2012. Núm. 162.

LEY 31/1990, de 27 de diciembre, de Presupuestos Generales del Estado para 1991. BOE de 28 de diciembre de 1990. Núm. 311.

Martín Valverde, A; Rodríguez Sañudo, F. y García Murcia, J.: Derecho del Trabajo, Editorial Tecnos, Madrid, 2009.

MoREAU, J.: Essai sur une politique de l'Economie Sociale, du troisième secteur vécu au troisième secteur voulu, C.I.E.M, Paris, 1982.

SCHUMPETER, J.A.: Teoría del desarrollo económico. FCE. México, 1976.

SIMONDE DE SISMONDI, J.C.L.: Nuevos principios de economía política. O de la riqueza en sus relaciones con la población, Editorial Icaria, Barcelona, 2016.

StUART MilL, J.: Principles of Political Economy with some of their Applications to Social Philosophy, Hackett Publishing, Indianapolis, 2004.

WALRAS, L.: Études d'économie sociale, Dentu, Paris, 1896. 